\title{
Control of Open Voltage of Organic Photovoltaic Cells Using Fluorinated Self-Assembled Monolayer
}

\author{
Tatsuo Mori, Xiaoling Ma*, Hideo Furuhashi and Takao Nishikawa** \\ Department of Electrical Engineering, Aichi Institute of Technology, \\ 1247 Yachigusa, Yakusa-cho, Toyota, Aichi 470-0392, Japan \\ *Department of Electrical Engineering and Computer Science, \\ Nagoya University, Furo-cho, Chikusa, Nagoya, Aichi 464-8603, Japan \\ **Center for Regional Collaboration in Research and Education, Iwate University \\ 4-3-5 Ueda, Morioka, Iwate, 020-8551, Japan \\ t2mori@aitech.ac.jp
}

\begin{abstract}
Since the introduction of fluorinated self-assembled monolayer (FSAM) as a buffer layer increases the work function of indium tin oxide (ITO), it is effective for increasing in open voltage. The open voltage of organic thin-film photovoltaic cell (OPV) depends on the difference between the highest occupied molecular orbital level of donor material and the lowest unoccupied molecular orbital level of acceptor material and the work function difference between positive and negative electrodes. Consequently, it is successful to increase the open voltage using FSAM-modified ITO.
\end{abstract}

Keywords : Organic thin-film photovoltaic cell, Self-assembled monolayer, Open voltage, Carrier ejection

\section{Introduction}

Although organic thin-film photovoltaic cell (OPV) have always been studied before 1980', their power conversation efficiency (PCE) was very low and lower than $1 \%[1]$. The generation mechanism of electricity was to use exciton dissociation due to electric field in the depletion layer of Schottky interface. At 1986, C. W. Tang proposed the bilayer structure consisting of copper phthalocyanine $(\mathrm{CuPc})$ and perylene derivative (PV)[2]. In this structure, $\mathrm{CuPc}$ and $\mathrm{PV}$ act as a donor and a acceptor. Carriers are generated from exciton dissociation due to energy difference between $\mathrm{CuPc}$ and $\mathrm{PV}$ at the interface. This is called a hetero junction structure. In the mechanism without electric field, it is important for higher PCE to increase carrier generation caused by the increase in interface area and to efficiently transport the dissociated carriers to the electrodes. The ideal structure is called a bulk hetero junction structure[3].

In general, the PCE of solar cells is described as the follow:
$P C E=\frac{J_{s c} \times V_{o c} \times F F}{P_{i n}}$,

where $J_{\text {sc }}, V_{\text {oc }}, \mathrm{FF}$ and $\mathrm{P}_{\text {in }}$ are short-circuit current density, open voltage, fill factor and input power, respectively. Therefore, the high-efficiency of solar cell can be achieved by the increase in $J_{\mathrm{sc}}, V_{\mathrm{oc}}$, and $\mathrm{FF}$. The increase in $J_{\mathrm{sc}}$ for OPV can be obtained by the increase in optical absorption region. On the other hand, the $V_{\mathrm{oc}}$ for OPV depends on the difference $\Delta E$ between the highest occupied molecular orbital (HOMO) level of donor material and the lowest unoccupied molecular orbital (LUMO) level of acceptor material. In addition, since the $V_{\text {oc }}$ for OPV also depends on the work function difference $\Delta \phi$ between positive and negative electrodes, the $V_{\text {oc }}$ is limited by the either smaller. In this paper, we discuss that the work function difference is enlarged by increasing the work function of stable anode, indium tin oxide due to the modification of self-assembled monolayer[4-7] instead of using instable metal with lower work function as the positive electrode. 


\section{Experimental}

We used poly(3,4-ethylenedioxythiophene): poly(styrenesulfonate) (PEDOT:PSS), copper Phthalocyanine (CuPc), fullulen-C60 (C60), 2,9dimethyl-4,7-diphenyl-1,10-phenanthroline (BCP), and 4,4'-bis(carbazol-9-yl)biphenyl (CBP) from Luminescence Technology. We used (3.3.3trifluoropropyl)- triethoxysilane (F3SAM) and (heptadecafluoro-1,1,2,2-tetrahydrodecyl) triethoxy silane (F10SAM) as a SAM material from Gelest, Inc. The chemical structures are shown in Fig. 1.

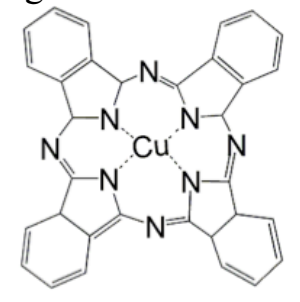

$\mathrm{CuPc}$



Fig. 1 Chemical structures of organic materials.

We purchased cleaned ITO substrates from Yamanaka Hutech Co. These substrates were cleaned by UV-ozone cleaner after ultrasonic cleaning in highly pure 2-propanol. Organic thin films were prepared on a glass substrate with an ITO transparent electrode (anode). The organic thin films were prepared using the vacuum deposition at a pressure of $0.8 \sim 1 \times 10^{-3} \mathrm{~Pa}$ at room temperature. We carried out a vacuum-deposition with EO-5 (EIKO Co.).

Absorption spectra were measured by spectrophotometer (U-3000, Hitachi Co., Ltd.). The work functions of ITO and FSAM-modified ITO are measured with the photoemission yield spectroscopy in air (AC-2, Riken Keiki Co.). Current-voltage characteristics were measured using a source measure unit (Keithley 2400 Source Measure Unit). Photocurrents were measured with the mask $\left(2 \times 2 \mathrm{~mm}^{2}\right.$, equal to device area) under a quasi-solar simulator of AM1.5, $100 \mathrm{~mW} / \mathrm{cm}^{2}$ (Seric Ltd., XIL-03E). The measurements of current-voltage characteristics were carried out under vacuum of $\sim 0.1 \mathrm{~Pa}$.

\section{Results and Discussions}

3.1. Work functions of FSAM on ITO substrates and application to $\mathrm{CuPc} / \mathrm{C} 60$ device

Although the ITO's surface is hydrophilic, the surface of FSAM on $\mathrm{Si}$ is hydrophobic and its contact angle is $\sim 110^{\circ}$ because of fluorine atoms[8]. Fig. 2 shows the photoelectron yield vs. photon energy of bare and FSAM-modified ITO substrates. While the work function of UV-ozone-cleaned bare ITO substrate is estimated to be $5.1 \mathrm{eV}$, those of F3SAM- or F10SAM-modified ITO substrates are estimated to be 5.4 and $5.6 \mathrm{eV}$, respectively. It is thought that the increase in work function is caused by the strong polarization of FSAM molecules. Ishii et al. have already reported the effect of interface dipole layer on the metal-organic interface[9,10]. The dipole moments of F3SAM and F10SAM molecules are calculated to be $2.51 \mathrm{D}$ and $2.89 \mathrm{D}$ using Scigress MO Compact, AM1, respectively.

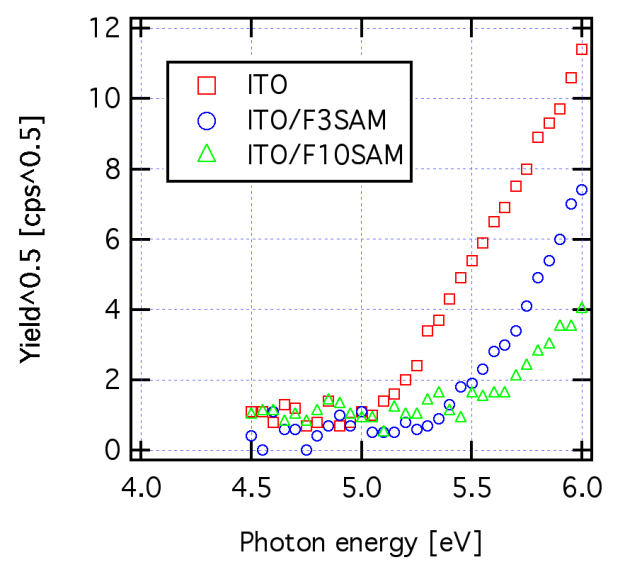

Fig. 2 The photoelectron yield vs. photon energy of bare and FSAM-modified ITO substrates.

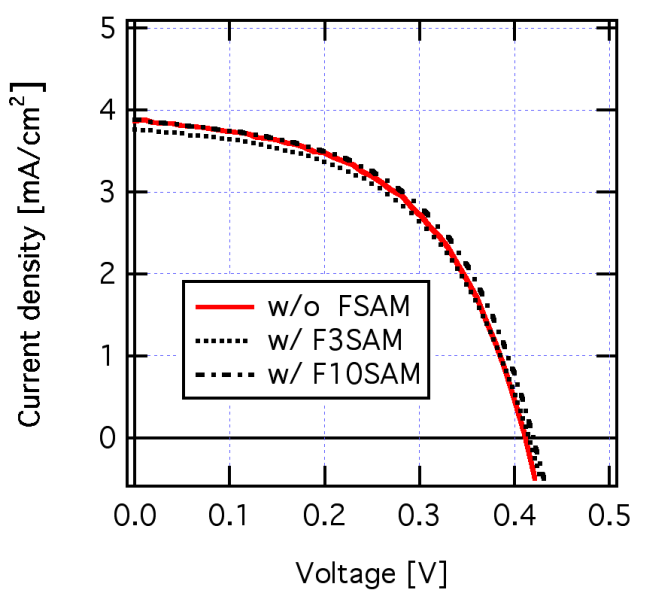

Fig. 3 The photocurrent vs. voltage for ITO/ w/o or w/ F3SAM or F10SAM/CuP(40nm)/C60(30nm) 
$/ \mathrm{BCP}(15 \mathrm{~nm}) / \mathrm{Al}$.

Table 1 PV properties of three above specimens.

\begin{tabular}{ccccc}
\hline specimen & $\begin{array}{c}J_{\text {sc }} \\
{\left[\mathrm{mA} / \mathrm{cm}^{2}\right]}\end{array}$ & $\begin{array}{c}V_{\mathrm{oc}} \\
{[\mathrm{V}]}\end{array}$ & FF & $\begin{array}{c}\text { PCE } \\
{[\%]}\end{array}$ \\
\hline $\begin{array}{c}\text { W/o } \\
\text { FSAM } \\
\text { W/ } \\
\text { F3SAM } \\
\text { W/ }\end{array}$ & 3.87 & 0.41 & 0.52 & 0.83 \\
F10SAM & 3.76 & 0.42 & 0.51 & 0.80 \\
\hline
\end{tabular}

Fig. 3 shows the photocurrent vs. voltage for ITO/ w/o or w/ F3SAM or F10SAM/CuP(40nm)/ C60 $(30 \mathrm{~nm}) / \mathrm{BCP}(15 \mathrm{~nm}) / \mathrm{Al}$. Table 1 shows the PV properties of three specimens. Three photocurrent curves are similar and there is no remarkable difference among three specimens. The $V_{\text {oc }}$ does not change by the modification of FSAM although the work function difference $\Delta \phi$ between positive and negative electrodes increases from $0.9 \mathrm{eV}$ to $1.4 \mathrm{eV}$ in Figs. $4 \mathrm{a}$ and $4 \mathrm{~b}$. Since the difference $\Delta E$ between the HOMO level of $\mathrm{CuPc}$ and the LUMO level of $\mathrm{C} 60$ is $0.5 \mathrm{eV}, V_{\mathrm{oc}}$ will be controlled by the smaller, $\Delta E$.
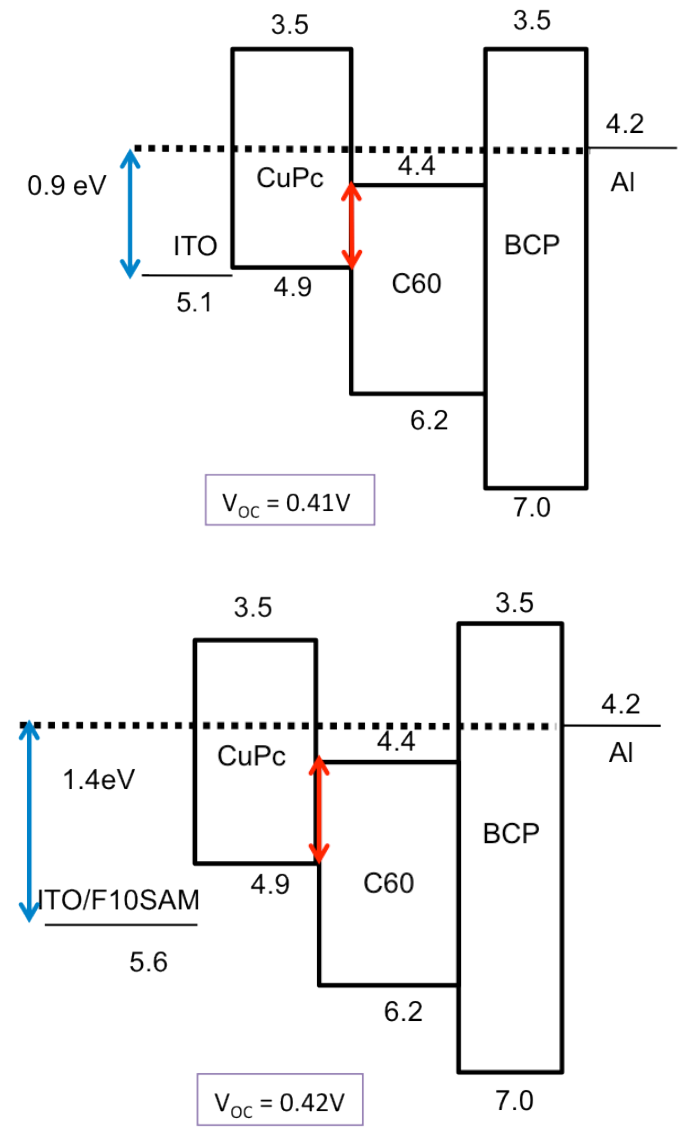

Fig. 4 The energy diagram of (a) OPV without FSAM (top) and (b) OPV with F10SAM(bottom).

\subsection{Application to $\mathrm{CBP} / \mathrm{C} 60$ device}

An alkyl chain is regarded as an insulation part because it's chemical structure is the same as polyethylene. Although we were deeply concerned about the carrier ejection from $\mathrm{CuPc}$ to ITO, we used two kinds of FSAM molecules with different alkyl chain. However, it is found that the carrier ejection can occur through the alkyl chain of FSAM.

Since the work function difference $\Delta \phi$ between positive and negative electrodes is larger than the difference $\Delta E$ between the HOMO level of donor material and the LUMO level of acceptor material, the $V_{\mathrm{oc}}$ does not change by the modification of FSAM. In this section, we will reduce the $\Delta \phi$ by using Ag electrode with higher work function, $4.7 \mathrm{eV}$. The $\Delta \phi$ of ITO-Ag system is estimated to be $0.4 \mathrm{eV}$. In addition, the $\Delta E$ will be extended by using the donor material CBP with deeper HOMO level, $5.9 \mathrm{eV}$. The $\Delta E$ of $\mathrm{CBP} / \mathrm{C} 60$ system is estimated to be $1.4 \mathrm{eV}$. Consequently, the $\Delta \phi$ of ITO-Ag system is smaller than the $\Delta E$ of $\mathrm{CBP} / \mathrm{C} 60$ system. However, the use of CBP lowers the optical absorption due to the expanding of energy band gap as shown in Fig. 5. CuPc has two absorption regions of $<400 \mathrm{~nm}$ and $550-800 \mathrm{~nm}$. CBP has the absorption in the wavelength region of $<400 \mathrm{~nm}$ because of the wide HOMO-LUMO energy gap of $3.3 \mathrm{eV}$. Therefore, the PCE of $\mathrm{CBP} / \mathrm{C} 60$ device will be reduced by decreasing $J_{\mathrm{sc}}$. This photocurrent is thought to be due to the absorption of $\mathrm{C} 60$.

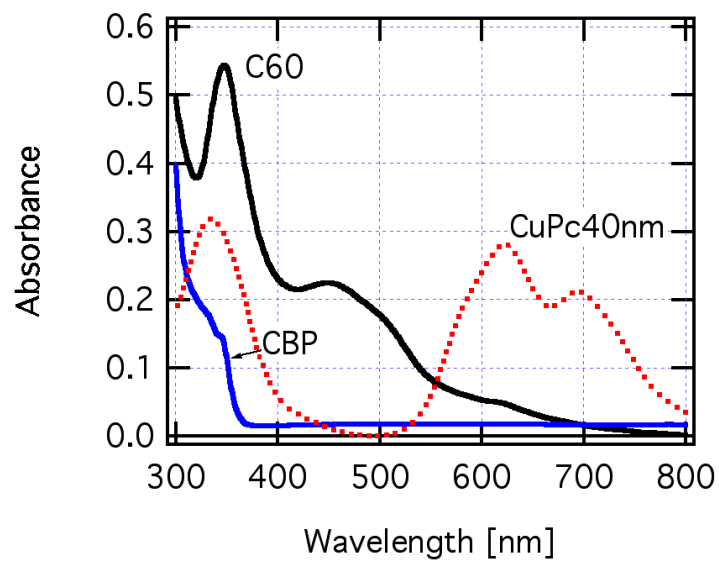

Fig. 5 The absorbance spectra of C60 (40nm), CBP $(10 \mathrm{~nm})$ and $\mathrm{CuPc}(40 \mathrm{~nm})$.

Fig. 6 shows the photocurrent vs. voltage for ITO/ w/o or w/ F3SAM or F10SAM/ $\mathrm{CBP}(10 \mathrm{~nm}) / \mathrm{C} 60$ (40nm)/BCP(15nm)/Ag. We tried to fabricate the device using thicker CBP layer to obtain comparatively higher absorption. Unfortunately, we could not fabricate the device 
showing a PV property. The PV properties of CBP/C60 devices are not good, and especially show a poor FF. The PCE of $\mathrm{CBP} / \mathrm{C} 60$ devices is maximum $0.20 \%$ and one fourth of the $\mathrm{CuPc} / \mathrm{C} 60$ devices. The $V_{\text {oc }}$ of the devices without or with F3SAM are lower than that of the device with F10SAM. This is thought to be due to the leakage current for the devices without or with F3SAM. In addition, the $J_{\mathrm{sc}}$ of the devices without or with F3SAM are lower than that of the devices with F10SAM. Therefore, it does not function adequately to eject holes from CBP to ITO or ITO/F3SAM.

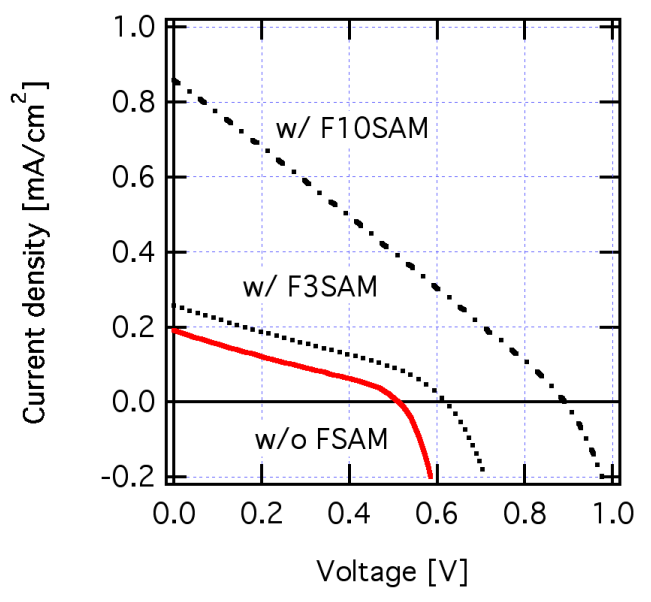

Fig. 6 The photocurrent vs. voltage for ITO/ w/o or w/ F3SAM or F10SAM/CBP(10nm) /C60(40nm) $/ \mathrm{BCP}(15 \mathrm{~nm}) / \mathrm{Ag}$.

Table $2 \mathrm{PV}$ properties of three specimens in Fig. 6.

\begin{tabular}{ccccc}
\hline specimen & $\begin{array}{c}J_{\text {sc }} \\
{\left[\mathrm{mA} / \mathrm{cm}^{2}\right]}\end{array}$ & $\begin{array}{c}V_{\text {oc }} \\
{[\mathrm{V}]}\end{array}$ & FF & $\begin{array}{c}\text { PCE } \\
{[\%]}\end{array}$ \\
\hline $\begin{array}{c}\text { w/o } \\
\text { FSAM } \\
\text { W/ }\end{array}$ & 0.19 & 0.50 & 0.31 & 0.03 \\
$\begin{array}{c}\text { F3SAM } \\
\text { W/ }\end{array}$ & 0.26 & 0.62 & 0.32 & 0.05 \\
F10SAM & 0.86 & 0.88 & 0.27 & 0.20 \\
\hline
\end{tabular}

As shown in Fig. 7, the work function difference $\Delta \phi, \quad 0.4 \mathrm{eV}$ is smaller than the $\mathrm{HOMO}_{\text {donor }}-\mathrm{LUMO}_{\text {acceptor }}$ difference, $\Delta E, 1.5 \mathrm{eV}$ for the CBP/C60 OPV without FSAM. If the $V_{\text {oc }}$ is limited by $\Delta \phi$, the $V_{\text {oc }}$ should be $0.4 \mathrm{~V}$. However, the experimental $V_{\text {oc }}$ is $0.50 \mathrm{~V}$. Although we used the value of ultra cleaned $\mathrm{Ag}$, the work function of vacuum-deposited $\mathrm{Ag}$ is estimated to be smaller than the value of ultra cleaned $\mathrm{Ag}[11]$. Therefore, the $\Delta \phi$ will become larger than the ideal value. But the Voc of the device without FSAM is controlled by the $\Delta \phi$. On the other hand, in the case of $\mathrm{CBP} / \mathrm{C} 60$ OPV with F10SAM, since the $\Delta \phi$ increases to $0.9 \mathrm{eV}$, the $V_{\text {oc }}$ is limited by $\Delta \phi, 0.9 \mathrm{~V}$ and is obtained to $0.88 \mathrm{~V}$. That is, the high work function due to FSAM-modification is effective to control $V_{\text {oc }}$ for OPV.
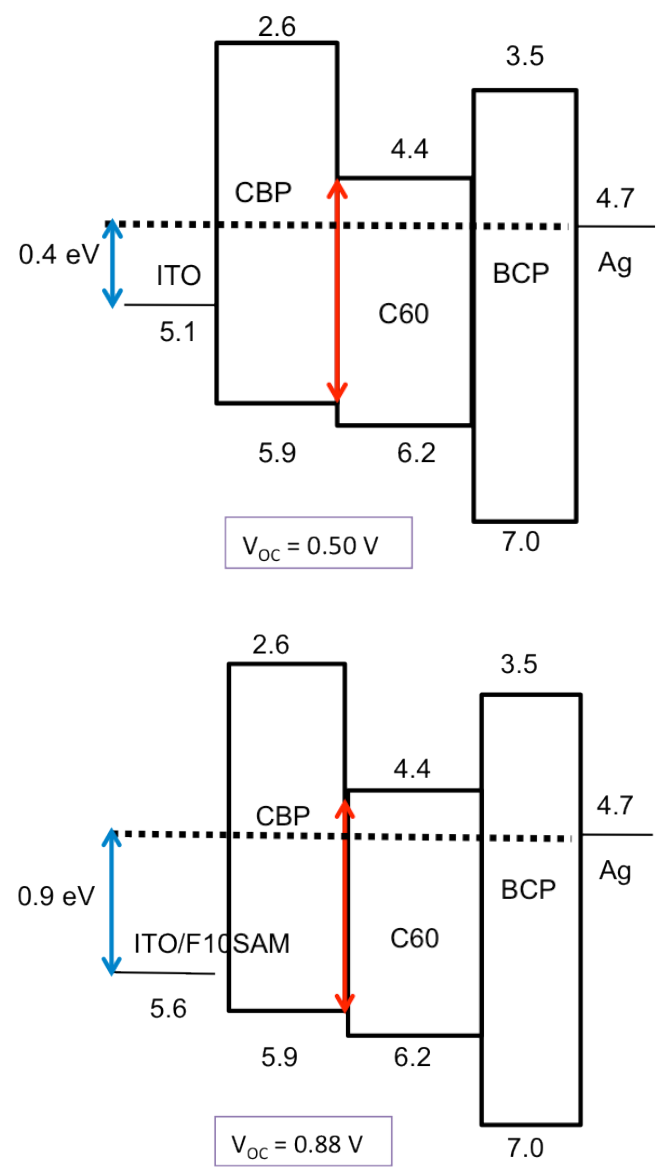

Fig. 7 The energy diagram of (a) CBP/C60 OPV without FSAM (top) and (b) $\mathrm{CBP} / \mathrm{C} 60 \mathrm{OPV}$ with F10SAM(bottom).

Although we use CBP with a large HOMO-LUMO gap as a donor material, CBP is not appropriate to a donor material for OPV. The donor material with a small HOMO-LUMO gap and a deep HOMO level must be used to obtain a high $J_{\text {sc }}, V_{\mathrm{oc}}$, and PCE.

\section{Conclusions}

We applied FSAM-modified ITO to OPV. In the case of large $\mathrm{HOMO}_{\text {donor }}-\mathrm{LUMO}_{\text {acceptor }}$ difference, the increase in $V_{\text {oc }}$ can be controlled by extending the work function difference between positive and negative electrodes. In general, the metals with deep work function are more expensive noble metal or have a high melting point. Therefore, to obtain deep work function due to 
FSAM-modification is more effective than to use instable metal with lower work function.

\section{Acknowledgements}

This research is partially obtained by a financial assistance of a MEXT-Supported Program for the Strategic Research Foundation at Private Universities (S1001033, 2010-2014) and the AIT Special Grant for Education and Research.

\section{References}

1. S.-S. Sun, N. S. Sariciftci, ed: Organic Photovoltaics: Mechanism, Materials and Device (CRC Press, New York, 2005).

2. C. W. Tang, Appl. Phys. Lett., 48, (1986) 183.

3. M. Hiramoto, H. Fujisawa, M. Yokoyama, Appl. Phys. Lett., 58, (1991) 1062.

4. T. Mori, S. Nishino, T. Nishikawa, S. Ogawa, Organic Electronics, 9 (2008) 63.
5. T. Mori, S. Nishino, T. Nishikawa, S. Ogawa, Jpn. J. Appl. Phys., 47 (2008) 455.

6. T. Mori, M. Imanishi and T. Nishikawa, Appl. Phys. Exp., 4 (2011) 071601.

7. T. Mori, M. Imanishi, T. Nishikawa, $J$. Photopolym. Sci. Technol., 25 (2012) 327.

8. S. Kobayashi, T. Nishikawa, T. Takenobu, S. Mori, T. Shimoda, T. Mitani, S. Shimotani, N. Yoshimoto, S. Ogawa, and Y. Iwase, Nat. Mater, 3 (2004) 317.

9. H. Ishi, K. Sugiyama, E. Ito, and K. Seki, $A d v$. Mater., 11 (1999) 605.

10. T. Satoh, M. Imanishi, T. Nishikawa, T. Mori, Jpn. J. Appl. Phys., 51 (2012) 035701.

11. C. J. Brabec, A. Cravino, D. Messner, N. S. Sariciftici, T. Fromherz, M. T. Rispens, L. Sanchez, J. Hummelen, Adv. Funct. Mater., 11 (2001) 374. 